
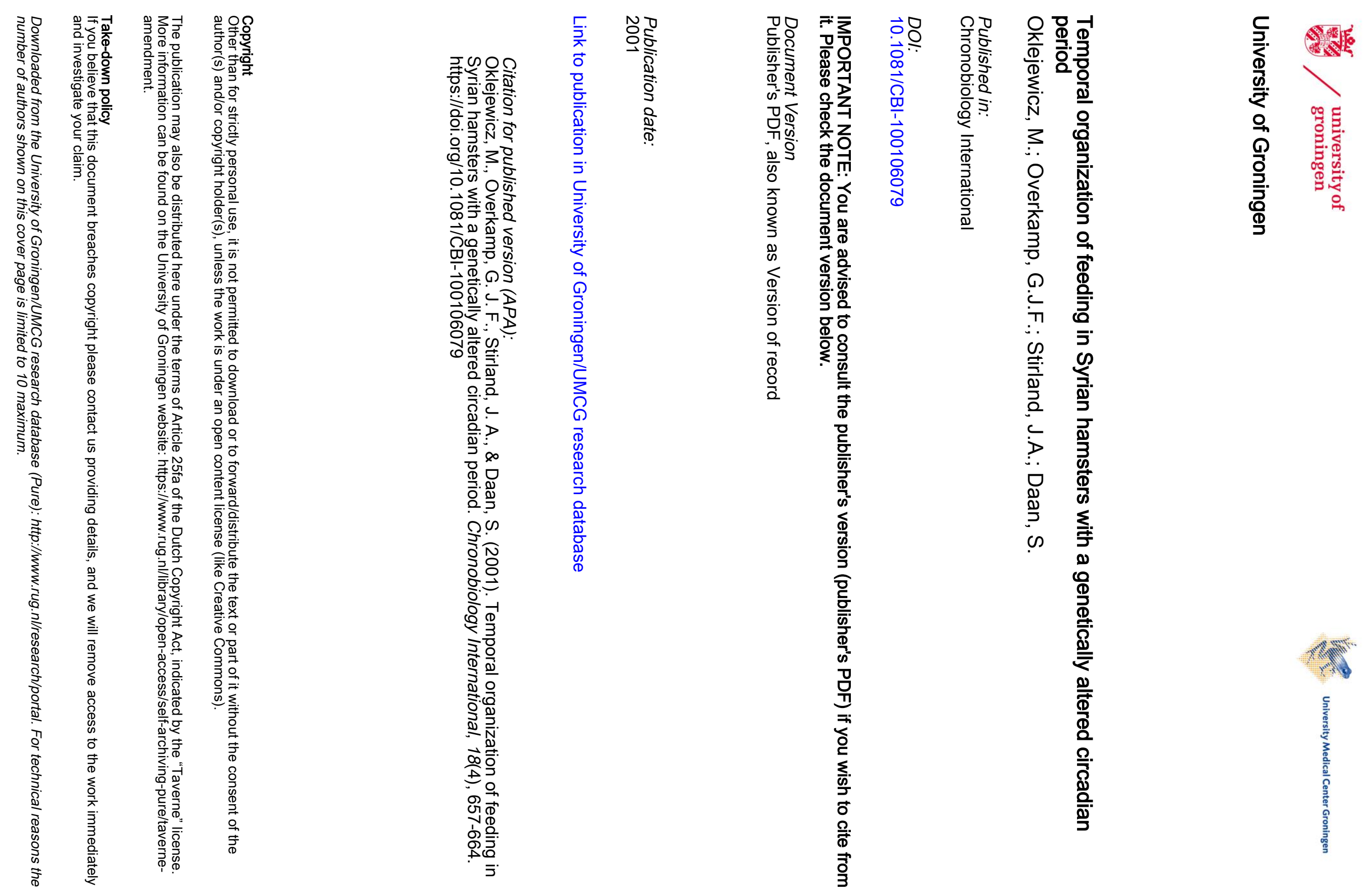
CHRONOBIOLOGY INTERNATIONAL, 18(4), 657-664 (2001)

\title{
TEMPORAL ORGANIZATION OF FEEDING IN SYRIAN HAMSTERS WITH A GENETICALLY ALTERED CIRCADIAN PERIOD
}

\author{
Malgorzata Oklejewicz, ${ }^{1, *}$ Gerard J. F. Overkamp, ${ }^{1}$ \\ J. Anne Stirland, ${ }^{2}$ and Serge Daan ${ }^{1}$ \\ ${ }^{1}$ Zoological Laboratory, University of Groningen, The Netherlands \\ ${ }^{2}$ School of Biological Sciences, University of Manchester, \\ United Kingdom
}

\begin{abstract}
The variation in spontaneous meal patterning was studied in three genotypes (tau +/+, tau +/- and tau -/-) of the Syrian hamster with an altered circadian period. Feeding activity was monitored continuously in 13 individuals from each genotype in constant dim light conditions. All three genotypes had on average six feeding episodes during the circadian cycle (about $20 \mathrm{~h}$ in homozygous tau mutants and $22 \mathrm{~h}$ in heterozygotes compared with $24 \mathrm{~h}$ in wild-type hamsters). Thus, homozygous tau mutant hamsters had significantly more feeding episodes per $24 \mathrm{~h}$ than wild types, and heterozygotes were intermediate. The average duration of feeding bouts (FBs) was indistinguishable (around 30 minutes) among the three genotypes, whereas the intermeal (IM) intervals were significantly shorter for homozygote tau mutant hamsters (99 minutes), intermediate for heterozygotes (116 minutes), and the longest for wild-type hamsters (148 minutes). Thus, the meal-to-meal duration was on average $25 \%$ shorter in homozygous tau mutants (16\% in heterozygous) than in wild-type hamsters. The reduction of the circadian period has a pronounced effect on short-term feeding rhythms and meal frequency in hamsters carrying the tau mutation. (Chronobiology International, 18(4), 657-664, 2001)
\end{abstract}

*Corresponding author. Malgorzata Oklejewicz, University of Groningen, Zoological Laboratory, P.O. Box 14, 9750 AA Haren, The Netherlands. Fax: +31 50363 2148; E-mail: m.oklejewicz@ biol.rug.nl 
Key Words: Circadian; Feeding bout; Intermeal interval; Syrian hamster; tau mutation

\section{INTRODUCTION}

Spontaneous food intake in rodents is usually characterized by a series of discrete feeding episodes or meals, separated by intermeal (IM) intervals. This organization of feeding is under homeostatic feedback control, and several models have been advanced to guide the unraveling of the control circuits (1). Liporegulatory mechanisms, gastrointestinal tract content, the central noradrenergic system, and the ventromedial hypothalamus through lesioning studies have been shown experimentally to be involved in the short-term regulation of feeding (24). On the other hand, feeding behavior is also embedded in the endogenous behavioral pattern controlled by the circadian pacemaker in the suprachiasmatic nuclei (SCN; 5-8). While homeostatic feedback allows for a corrective control mechanism, circadian control provides for anticipatory adjustment to environmental change. The contribution of both to the organization of feeding behavior is underscored by the effect of complete SCN lesions, which disrupts the temporal organization of feeding yet leaves total food intake unaffected (9).

The aim of the present study was to describe the spontaneous feeding pattern in the Syrian hamster and to elucidate the contribution of the circadian pacemaker to the temporal organization of feeding. In particular, we were interested in defining the effect of an acceleration of the circadian cycle due to a single locus change in the Syrian hamster genome (10). In the homozygous tau mutant, the endogenous circadian cycle of locomotor activity is about $20 \mathrm{~h}$, a $17 \%$ reduction compared to the wild-type cycle of $24 \mathrm{~h}$. In this study, the spontaneous feeding activity was recorded in the three genotypes of hamster (homozygous tau mutants, heterozygotes with a circadian period of approximately $22 \mathrm{~h}$, and wildtype hamsters). If the circadian system controls the short-term organization of feeding, the feeding cycle in mutant hamsters is expected to shorten by decreasing the duration of feeding and/or the IM interval.

\section{MATERIALS AND METHODS}

Male Syrian hamsters (Mesocricetus auratus) were bred at the Zoological Laboratory, Haren, The Netherlands. Wild-type hamsters $(\operatorname{tau}+/+)$ were paired with homozygous tau mutants (tau $-/-)$ to produce heterozygous (tau $+/-)$ mutants. Crosses of tau $+/-$ hamsters were then set up to generate the experimental animals $(\operatorname{tau}+/+$, tau $+/-$, and tau $-/-)$. Breeding was performed under constant light conditions and ambient temperature $\left(23^{\circ} \mathrm{C} \pm 2^{\circ} \mathrm{C}\right)$ with food and water available ad libitum. General locomotor activity was monitored under constant 
dim red light (less than 0.5 lux) for 10 days to determine the circadian phenotype of the hamsters.

At 10 weeks of age, experimental male hamsters (13 individuals from each genotype) were transferred to individual recording cages $(25 \times 25 \times 40 \mathrm{~cm})$ in a temperature controlled $\left(23^{\circ} \mathrm{C} \pm 0.5^{\circ} \mathrm{C}\right)$ room. The room was continuously illuminated by dim red light (Philips, $36 \mathrm{~W} / 15$ red tube lights) with intensities from 0.1 to 0.5 lux. The experiment was carried out from October to December. Wood shavings were used as bedding material; food and water were available ad libitum and checked at random times once per week.

A passive infrared detector placed on top of the entry to the feeding tube recorded feeding activity. The feeding tube was separated from the rest of the cage by a Plexiglas wall and connected via a wire mesh tunnel $(5.5 \mathrm{~cm}$ diameter and $3 \mathrm{~cm}$ long). This allowed exclusive recording of feeding activity inside the feeding tube. Feeding was recorded automatically in 2-minute time bins by a recording system based on the personal computer (ERS).

For each hamster, 10 days of feeding activity were analyzed (examples are shown in Fig. 1). All nonfeeding intervals longer than 400 minutes represented the resting phases of the daily cycle. These were clearly distinguishable from IM intervals during the activity phase (Fig. 2) and were omitted from the analysis. A 20-minute IM criterion (e.g., 11,12) was applied to the data as follows: Any 2-minute time bins with feeding separated by fewer than 10 bins without feeding were considered to belong to the same meal or feeding bout (FB). Single time bins with feeding activity separated by more than 10 bins from the prior and the next time bins with feeding were discarded. Most of these cases occurred when the hamster occasionally visited the feeding tube without eating.

Medians of individual FB and IM interval durations were tested for statistical significance among genotypes by one-way analysis of variance (ANOVA)
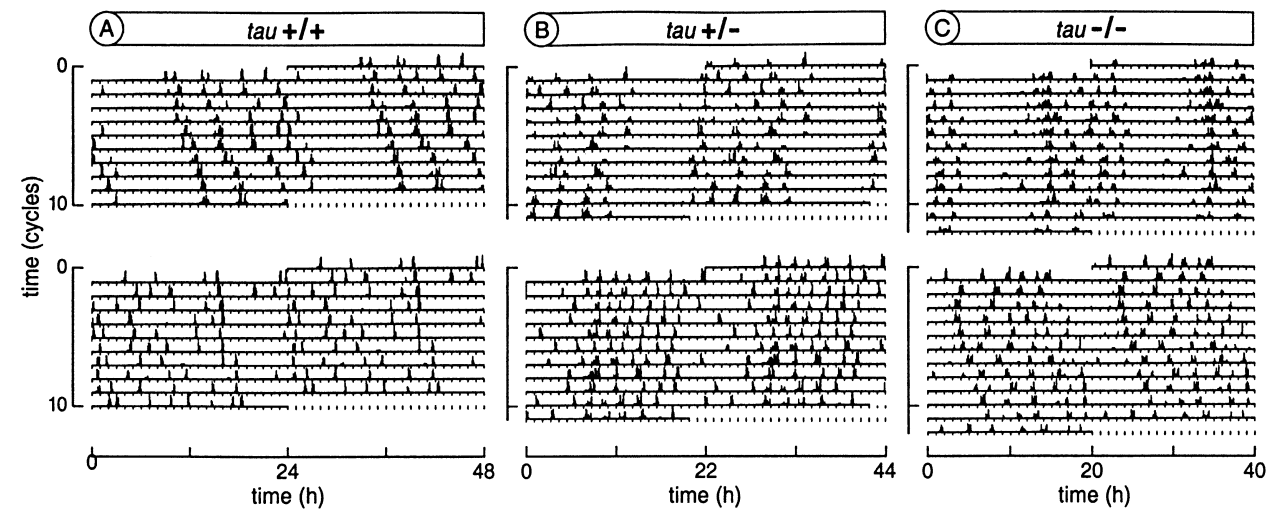

Figure 1. Examples of feeding activity recordings for two individuals from each genotype. The quantitative data are double plotted on a $24 \mathrm{~h}$ scale for wild-type hamsters $(\mathrm{A} ; \operatorname{tau}+/+)$, on $22 \mathrm{~h}$ for heterozygous hamsters (B; tau $+/-)$, and on $20 \mathrm{~h}$ for homozygous tau mutant hamsters (C; tau $-/-)$. 

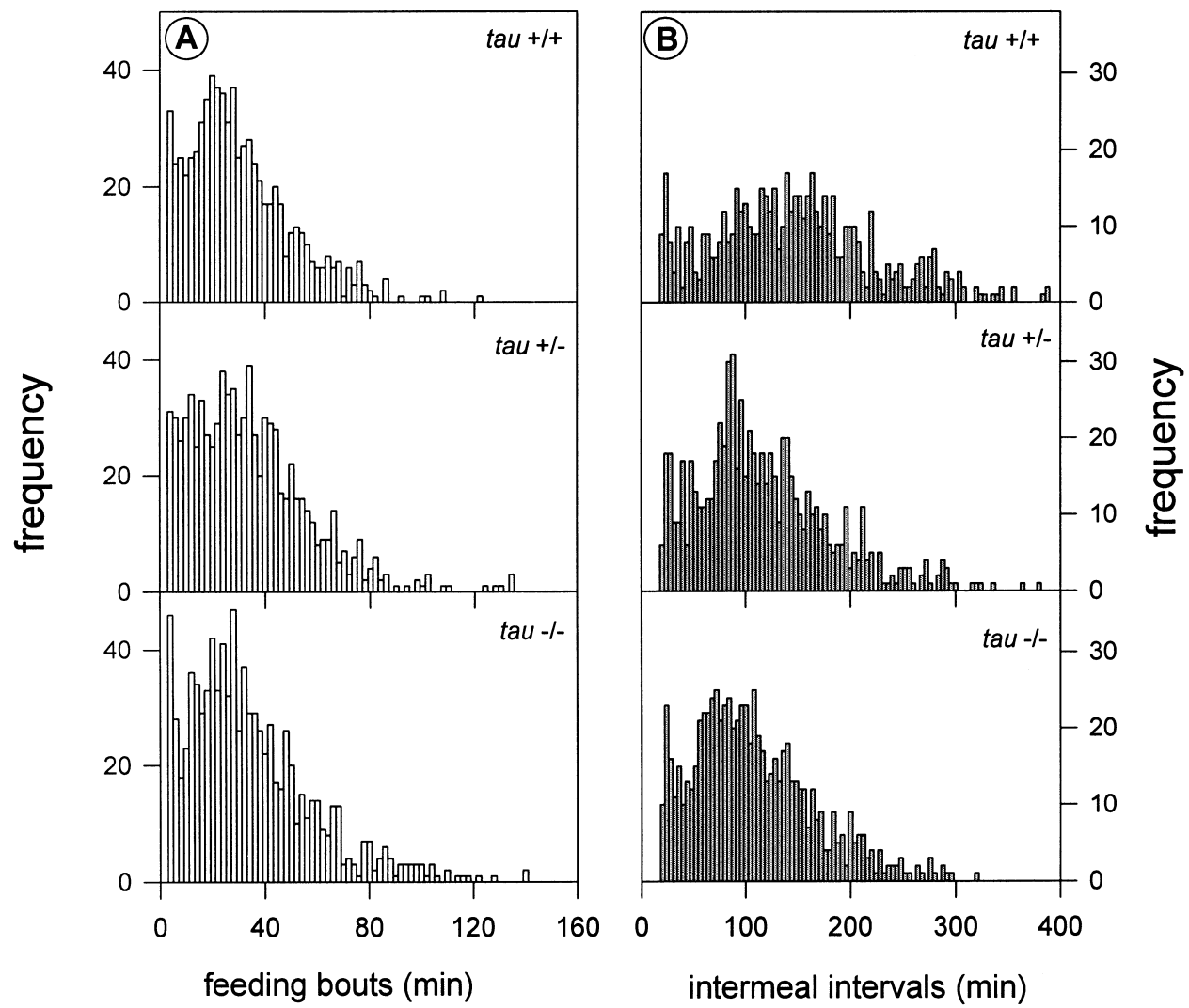

Figure 2. Frequency distribution of (A) all feeding bouts and (B) intermeal interval for wildtype $(\operatorname{tau}+/+)$, heterozygous (tau $+/-)$, and homozygous tau mutant hamsters (tau $-/-)$.

and post hoc Tukey test. The rhythmicity in feeding activity was calculated by chi-square periodogram time series analysis (13). All data are presented as means plus or minus the standard errors unless stated otherwise. Significance was accepted at $P<.05$ (two tailed).

\section{RESULTS}

All hamsters showed a robust circadian rhythm of feeding activity (Fig. 1). Food intake clustered into clear FBs separated by shorter IM intervals and longer (more than 400 minutes) nonfeeding episodes (Fig. 2). Despite exposure throughout their life only to constant illumination, about $80 \%$ of hamsters had a sharp onset of daily feeding, as presented in Fig. 1, and none of the hamsters became arrhythmic. The circadian period of feeding activity ranged from $23.8 \mathrm{~h}$ to $24.9 \mathrm{~h}$ for tau $+/+$ (mean $\pm \mathrm{SD} ; 24.5 \mathrm{~h} \pm 0.3 \mathrm{~h}$ ), from $21.4 \mathrm{~h}$ to $22.0 \mathrm{~h}$ for $t a u+/-$ hamsters $(21.8 \mathrm{~h} \pm 0.2 \mathrm{~h})$, and from $20.0 \mathrm{~h}$ to $20.7 \mathrm{~h}$ for $\operatorname{tau}-/-(20.3 \mathrm{~h} \pm 0.2 \mathrm{~h})$. 
The number of FBs per 24h varied significantly among the three genotypes $(P<.01$, ANOVA; Table 1$)$. When expressed per circadian cycle tau $+/+$, tau $+/-$, and tau $-/-$ had similar numbers of FBs $(P=.6$, ANOVA; Table 1$)$. A total of 2463 FBs was recorded over 10 days for the three genotypes (from $n=13$ hamsters per genotype), and their durations are presented in Fig. 2A. The peaks of the distributions for the three genotypes are similar. The individual medians of FBs were not statistically different among genotypes ( $P=.4$, ANOVA; Table 1$)$, and on average, all hamsters fed for about 30 minutes during a single FB.

The individual medians of IM intervals were significantly longer for the tau $+/+$ genotype than for the mutant hamsters $(P<.001$, ANOVA; Fig. 2B). Tau $+/-$ hamsters had intermediate IM intervals, which did not significantly differ from those of tau $-/-$ hamsters $(P>.05$, Tukey test). In addition to this analysis, we also calculated IM intervals with the criteria of 5 and 7 bins to test whether the chosen 10-bin criterion (see Material and Methods) affected our conclusions. The difference in IM intervals among genotypes with both 5 and 7 bins was still highly significant $(P<.001$, ANOVA). The mean values decreased by 3 and 6 minutes for tau $+/+$ and tau $-/-$ hamsters, respectively, with the criteria of 5 time bins.

The feeding cycle defined as the meal-to-meal interval reflects the difference in the duration of IM intervals among genotypes. Total time spent feeding reflects both the frequency and the duration of meals. Tau -/- hamsters spent significantly more time on feeding per $24 \mathrm{~h}$ than tau $+/+$ hamsters $(P<.0002$, ANOVA; Table 1). Heterozygotes were intermediate, but did not significantly differ from either homozygote $(P>.05$, Tukey test). In contrast, the total feeding time per circadian cycle was not statistically different among the genotypes $(P=$ .25 , ANOVA; Table 1).

\section{DISCUSSION}

The mutation of the circadian period in tau mutant hamsters has clear consequences on the organization of the feeding pattern. The duration of a single

Table 1. Characteristics of Feeding Patterns (Mean \pm SEM) for Three Genotypes of Hamsters

\begin{tabular}{lrrr}
\hline & tau $+/+$ & tau $+/-$ & tau $-/-$ \\
\hline Number of feeding bouts per 24h & $5.6(0.2)$ & $6.5(0.4)$ & $6.9(0.3)^{\mathrm{a}}$ \\
Number of feeding bouts per circadian cycle & $5.6(0.2)$ & $6.0(0.4)$ & $5.8(0.3)$ \\
Feeding bout (minutes) & $28.1(2.4)$ & $31.3(1.8)$ & $30.8(1.4)$ \\
Intermeal interval (minutes) & $148.0(7.6)$ & $115.6(8.4)$ & $99.5(3.7)^{\mathrm{a}}$ \\
Feeding cycle (h) & $2.9(0.2)$ & $2.4(0.2)$ & $2.2(0.1)^{\mathrm{a}}$ \\
Total feeding time $\left(\mathrm{h} \cdot 24 \mathrm{~h}^{-1}\right)$ & $2.8(0.2)$ & $3.7(0.2)$ & $4.0(0.2)^{\mathrm{a}}$ \\
Total feeding time $\left(\mathrm{h} \cdot\right.$ cycle $\left.^{-1}\right)$ & $2.8(0.2)$ & $3.1(0.1)$ & $2.9(0.1)$ \\
\hline
\end{tabular}

${ }^{\mathrm{a}}$ Significant variation among genotypes, $P<.05$. 
meal was similar for all circadian phenotypes, but the time between meals was shortened on average by $33 \%$ in homozygote mutants and by $22 \%$ in heterozygote tau mutants compared with wild-type hamsters.

It is well established that the organization of spontaneous short-term feeding serves the energetic needs of the organism. The short-term feeding and activity rhythms have been extensively studied in strictly ultradian organized herbivorous microtine rodents, characterized by diurnal as well as nocturnal feeding $(14,15)$. The ultradian organization of physiology and behavior in these species is equally pronounced in light-dark cycles and in continuous illumination. In Syrian hamsters exposed to continuous lighting conditions throughout their life to evoke more pronounced ultradian organization of feeding behavior (16), all genotypes of hamster had feeding bouts organized within the subjective night. Further, in comparison with Microtus species, Syrian hamsters showed a strong circadian organization of feeding behavior that was not damped out in prolonged constant conditions.

The temporal organization of feeding in the three hamster genotypes reflects the energetic requirements. Homozygous and heterozygous tau mutant genotypes have a higher average metabolic rate (oxygen consumption per 24h) compared with wild-type hamsters $(17,18)$. Thus, they would be expected to have higher overall rates of food intake. If the intake during feeding is the same across genotypes, we expect tau mutants to spend more time feeding. Indeed, the daily feeding time was longer in the tau mutant than in wild-type hamsters. At the same time, the metabolic rate per circadian cycle was not distinguishable among the three genotypes, which corresponds well with the similar duration of feeding per circadian cycle reported in the present study.

Effects of circadian period mutations on high-frequency rhythms have previously been demonstrated in Drosophila per mutants (19). In the tau mutant hamster, Loudon et al. (20) showed that the luteinizing hormone and cortisol interpulse intervals were longer than in wild-type hamsters rather than shorter. Electrophysiological measurements of ultradian rhythmicity within the circadian pacemaker revealed no difference between genotypes in periods in the ultradian range (21). Both results are in contrast with our results on the feeding cycle.

The present study demonstrates that the change in frequency of circadian oscillations modifies the timing of onset of short-term feeding cycles in hamsters carrying the tau mutation by about $25 \%$ in tau $-/-$ and by about $16 \%$ in tau +/- hamsters compared with wild-type hamsters. This suggests that, in addition to its control over circadian organization of feeding behavior, the circadian pacemaker modifies the ultradian timing of food intake in the Syrian hamster.

\section{ACKNOWLEDGMENT}

We thank I. Pen, A. Strijkstra, and R. Hut for discussions and continued support and Dick Visser for preparing the actogram figure. The study was per- 
formed under license DEC 2117\#5 from the Animal Experimentation Committee, University of Groningen.

\section{REFERENCES}

1. Toates, F.M. Control of food intake by energy supply. Nature 1974, 251, 710-711.

2. Balagura, S.; Devenport, L.D. Feeding pattern of normal and ventromedial hypothalamic lesioned male and female rats. J. Comp. Physiol. Psychol. 1970, 71, 357364.

3. Le Magnen, J. The metabolic bases of dual periodicity of feeding in rats. Behav. Brain Sci. 1981, 4, 561-607.

4. Richter, C.P.; Epstein, A.N. Control of meal size by central noradrenergic action. Proc. Natl. Acad. Sci. USA 1975, 72, 3740-3743.

5. Stephan, F.K.; Zucker, I. Circadian rhythms in drinking behavior and locomotor activity are eliminated by hypothalamic lesion. Proc. Natl. Acad. Sci. USA 1972, 69, 1583-1586.

6. Stephan, F.K.; Nunez, A.A. Elimination of circadian rhythms in drinking, activity, sleep and temperature by isolation of the suprachiasmatic nuclei. Behav. Biol. 1977, 20, 1-16.

7. Nagai, K.T.; Nishio, H.; Nakagawa, S.; Nakamura, S.; Fukuda, Y. Effect of bilateral lesions of the suprachiasmatic nuclei on the circadian rhythm of food-intake. Brain Res. 1978, 142, 348-389.

8. Van den Pol, A.N.; Powley, T. A fine-grained anatomical analysis of the role of the rat suprachiasmatic nucleus in circadian rhythms of feeding and drinking. Brain Res. 1979, 160, 307-326.

9. Nakagawa, H.; Nagai, K.; Kida, K.; Nishio, H. Control mechanism of circadian rhythms of feeding behavior and metabolism influenced by food intake. In Biological Rhythms and Their Central Mechanism; Suda, M., Hayaishi, O., Nakagawa, H., Eds.; Elsevier Press: New York, 1979; 283-294.

10. Ralph MR, Menaker M. A mutation of the circadian system in golden hamsters. Science 1988, 241, 1225-1227.

11. Mrosovsky, N.; Boshes, M. Meal patterns in dormice. Appetite 1986, 7, 177-186.

12. Eckel, L.A.; Houpt, T.A.; Geary, N. Spontaneous meal patterns in female rats with and without access to running wheels. Physiol. Behav. 2000, 70, 397-405.

13. Sokolove, P.G.; Bushel, W.N. The chi square periodogram: its utility for analysis of circadian rhythms. J. Theor. Biol. 1978, 72, 131-160.

14. Daan, S.; Slopsema, S. Short-terms rhythms in foraging behaviour of the common vole, Microtus arvalis. J. Comp. Physiol. 1978, 127, 215-227.

15. Gerkema, M.; Daan, S. Ultradian rhythms in behavior: the case of the common vole. In Ultradian Rhythms in Physiology and Behavior; Schulz, H., Lavie, P., Eds.; Exp. Brain Res. 1985, Suppl. 12, 11-31.

16. Honma, K.; Hiroshige, T. Endogenous ultradian rhythms in rats exposed to prolonged continuous light. Am. J. Physiol. 1978, 235, 250-256.

17. Oklejewicz, M.; Hut, R.A.; Daan, S.; Loudon, A.S.I.; Stirland, A.J. Metabolic rate changes proportionally to circadian frequency in tau mutant Syrian hamsters. J. Biol. Rhythms 1997, 12, 413-422. 
18. Oklejewicz, M.; Hut, R.A.; Daan, S. Effect of deuterium on the circadian period and metabolism in wild-type and tau mutant Syrian hamsters. Physiol. Behav. 2000, 71, 69-74.

19. Kyriacou, C.P. The molecular ethology of the period gene in Drosophila. Behav. Genet. 1990, 20, 191-211.

20. Loudon, A.S.I.; Wayne, N.L.; Krieg, R.; Iranmanesh, A.; Veldhuis, J.D.; Menaker, M. Ultradian endocrine rhythms are altered by a circadian mutation in the Syrian hamster. Endocrinology 1994, 135, 712-718.

21. Yamazaki, S.; Kerbeshian, M.C.; Hocker, C.G.; Block, G.D.; Menaker, M. Rhythmic properties of the hamster suprachiasmatic nucleus in vivo. J. Neurosci. 1998, 18, 10709-10723.

Received January 17, 2001

Revised February 12, 2001

Accepted March 15, 2001 